\title{
Influence of pretreatment growth rate on Gamma Knife treatment response for vestibular schwannoma: a volumetric analysis
}

\author{
Patrick P. J. H. Langenhuizen, MSc, ${ }^{1,2}$ Svitlana Zinger, PhD, ${ }^{2}$ Patrick E. J. Hanssens, MD, ${ }^{1}$ \\ Henricus P. M. Kunst, MD, PhD, ${ }^{3}$ Jef J. S. Mulder, MD, PhD, ${ }^{3}$ Sieger Leenstra, MD, PhD, ${ }^{4}$ \\ Peter H. N. de With, PhD, ${ }^{2}$ and Jeroen B. Verheul, MD, PhD ${ }^{1}$ \\ ${ }^{1}$ Gamma Knife Center Tilburg, Department of Neurosurgery, ETZ Hospital, Tilburg; ${ }^{2}$ Eindhoven University of Technology, \\ Eindhoven; ${ }^{3}$ Department of Otolaryngology, Radboud Institute of Health Sciences, Radboud University Medical Center, \\ Nijmegen; and ${ }^{4}$ Department of Neurosurgery, Erasmus Medical Center, Rotterdam, The Netherlands
}

\begin{abstract}
OBJECTIVE The aim of this study was to gain insight into the influence of the pretreatment growth rate on the volumetric tumor response and tumor control rates after Gamma Knife radiosurgery (GKRS) for incidental vestibular schwannoma (VS).

METHODS All patients treated with GKRS at the Gamma Knife Center, ETZ Hospital, who exhibited a confirmed radiological progression of their VS after an initial observation period were included. Pre- and posttreatment MRI scans were volumetrically evaluated, and the volume doubling times (VDTs) prior to treatment were calculated. Posttreatment volumes were used to create an objective mathematical failure definition: 2 consecutive significant increases in tumor volume among 3 consecutive follow-up MRI scans. Spearman correlation, Kaplan-Meier survival analysis, and Cox proportional hazards regression analysis were used to determine the influence of the VDT on the volumetric treatment response.
\end{abstract}

RESULTS The resulting patient cohort contained 311 patients in whom the VDT was calculated. This cohort had a median follow-up time of 60 months after GKRS. Of these 311 patients, 35 experienced loss of tumor control after GKRS. The pretreatment growth rate and the relative volume changes, calculated at 6 months and 1, 2, and 3 years following treatment, showed no statistically significant correlation. Kaplan-Meier analysis revealed that slow-growing tumors, with a VDT equal to or longer than the median VDT of 15 months, had calculated 5- and 10-year control rates of $97.3 \%$ and $86.0 \%$, respectively, whereas fast-growing tumors, with a VDT less than the median growth rate, had control rates of $85.5 \%$ and $67.6 \%$, respectively (log-rank, $p=0.001$ ). The influence of the VDT on tumor control was also determined by employing the Cox regression analysis. The resulting model presented a significant $(p=0.045)$ effect of the VDT on the hazard rates of loss of tumor control.

CONCLUSIONS By employing a unique, large database with long follow-up times, the authors were able to accurately investigate the influence of the pretreatment VS growth rate on the volumetric GKRS treatment response. The authors have found a predictive model that illustrates the negative influence of the pretreatment VS growth rate on the efficacy of radiosurgery treatment. The resulting tumor control rates confirm the high efficacy of GKRS for slow-growing VS. However, fastgrowing tumors showed significantly lower control rates. For these cases, different treatment strategies may be considered. https://thejns.org/doi/abs/10.3171/2018.6.JNS18516

KEYWORDS acoustic neuroma; Gamma Knife radiosurgery; pretreatment growth rate; treatment failure definition; vestibular schwannoma; volumetric tumor response; stereotactic radiosurgery

$\mathrm{G}$ AmMA Knife radiosurgery (GKRS) has proven to be an effective treatment strategy for vestibular schwannoma (VS)., 1,2,13,23 However, when these tumors are treated without documented growth, the stability observed after radiosurgery can be mistaken for a radiosurgical effect in those tumors that would have been stable in their natural course. In other words, radiosurgical efficacy in terms of true tumor control can only be accurately evaluated in patients who have exhibited radiological progression prior to treatment. ${ }^{10}$ Nevertheless,

ABBREVIATIONS GKRS = Gamma Knife radiosurgery; VDT = volume doubling time; VS = vestibular schwannoma.

SUBMITTED February 28, 2018. ACCEPTED June 12, 2018.

INCLUDE WHEN CITING Published online November 16, 2018; DOI: 10.3171/2018.6.JNS18516. 
reporting is sparse on the efficacy of stereotactic radiosurgery in patient cohorts with proven tumor growth. ${ }^{8,9,14,17,21}$ Moreover, only a few studies have addressed pretreatment growth rate as a potential prognostic factor for the GKRS treatment outcome. ${ }^{9,14,21}$ Recently, Marston et al. indicated that fast-growing VSs are more likely to continue to grow after GKRS. ${ }^{9}$ However, other studies did not confirm this effect. . $^{8,1721}$

The conflicting results of these previous studies can possibly be explained by methodological imperfections such as limited patient numbers, 2D tumor measurements, insufficient follow-up times, and inconsistencies among treatment failure definitions. ${ }^{6} 16$ Any meaningful analysis of the effect of pretreatment growth rates on the GKRS treatment outcome should include a sufficient number of cases in which tumor control was not achieved. The low number of failures after GKRS and their possible late occurrence implies that such studies should include large patient numbers and long follow-up times. Furthermore, Varughese et al. determined that pretreatment growth rates can best be modeled by employing an exponential model to calculate volume doubling times (VDTs). ${ }^{20}$ Hence, volumetric measurements should be obtained. In addition, these measurements are also necessary to accurately determine posttreatment tumor progression., ${ }^{7}$

The possible influence of pretreatment growth rates on the radiosurgical outcome can be important for clinical decision-making. A pronounced effect of the pretreatment growth rate, with faster-growing tumors exhibiting lower control rates after GKRS, might be an argument for a different treatment strategy in these cases.

The objective of this study was to gain insight into the efficacy of GKRS in growing VSs and to provide information on the possible effect of the pretreatment growth rate on the treatment efficacy. To this end, we used data from a large patient cohort with proven pretreatment tumor progression and sufficient follow-up times after treatment, we performed volumetric measurements to accurately determine tumor sizes, and we propose an objective measure of treatment failure based on volumetric tumor progression.

\section{Methods}

The medical ethics review committee waived a formal approval procedure for this retrospective study. The study is compliant with the Dutch Medical Treatment Agreement Act.

\section{Patient Cohort}

In our prospectively collected database, all patients with unilateral VS treated with GKRS and a minimum follow-up of 2 years were identified. This cohort consisted of all VS patients remaining after excluding patients with neurofibromatosis type 2 , and those previously treated for their VS. All included patients showed a confirmed radiological progression of the tumor after an initial observation period. We excluded patients whose only pretreatment MRI study was obtained less than 6 months prior to treatment to reduce the impact of short observation periods resulting in misleadingly large growth rates. ${ }^{20}$ Patients were excluded if pretreatment MR images were unobtainable, inadequate (slice thickness $>2.5 \mathrm{~mm}$ ), or incompatible for accurate volumetric analysis.

\section{Pretreatment Growth Rate}

Pretreatment MR images obtained in patients included in this study were retrieved to assess the growth rate of each VS. This rate was calculated according to the VDT model proposed by Varughese et al..$^{20,21}$ This exponential model implies that tumor volumes double every set number of months and is calculated using the following formula: $V D T=\log (2) \times\left(T_{\text {treatment }}-T_{\text {pretreatment }}\right) /\left(\log \left[V_{\text {treatment }}\right]\right.$ $\left.-\log \left[V_{\text {pretreatment }}\right]\right)$. In this equation $T_{\text {treatment }}$ and $T_{\text {pretreatment }}$ represent the dates of the treatment and pretreatment MRI sessions, respectively. $V_{\text {treatment }}$ and $V_{\text {pretreatment }}$ are the corresponding volumes. As an example calculation, consider a tumor volume of $1 \mathrm{~cm}^{3} 1$ year before treatment and 2 $\mathrm{cm}^{3}$ at treatment. The result is a VDT of 12 months. The pretreatment tumor volumes were determined using GammaPlan (versions 10 and 11, Elekta AB) on T1-weighted contrast-enhanced MR images. If such MR images were unavailable or of poor quality, thin-slice T2-weighted MR images were used.

\section{Treatment and Follow-Up}

GKRS was performed using either Leksell Gamma Knife model 4C or Perfexion (since November 2008; both Elekta AB). A dose of $13 \mathrm{~Gy}$ was prescribed to the isodose line covering $90 \%$ (until May 2011) or 99\% (since May 2011) of the tumor volume. Treatment data such as beamon times, number of isocenters, dose-volume histograms, and Paddick conformity indices were collected.

After treatment, follow-up imaging was performed within a standard interval of 1 year using T1-weighted contrast-enhanced MRI with a slice thickness of $1 \mathrm{~mm}$. The follow-up interval was shortened in case of suspected radiological progression or new or worsening symptoms. If the tumor displayed radiological regression or stability for several years, the follow-up interval was extended. Volumetric tumor measurements were performed on all follow-up scans using GammaPlan (versions 10 and 11).

All patient records and volumetric tumor responses were reviewed to assess treatment failure. Loss of tumor control was always confirmed by the radiosurgical team, based on linear measurements. During the first 2 years after treatment, an increase in tumor volume was accepted and considered pseudoprogression, unless tumor expansion was deemed too excessive. Tumor growth after this period was deemed a failure. In addition, we looked for potentially missed failures, i.e., discrete volume increases undetected by linear measurements employed in the clinical setting, but detectable with the volumetric analysis performed for this research. For this purpose, a so-called volumetric failure was defined as 2 consecutive significant increases in tumor volume among 3 consecutive followup MRI sessions, where a minimum of $10 \%$ increase in volume is deemed significant. This value was based on the interobserver variability. To exclude changes in volume caused by pseudoprogression, only MR images obtained after the first 2 years of follow-up were used. 


\section{Correlation Between Pretreatment Growth Rate and GKRS Response}

To determine whether the pretreatment growth rate and the GKRS treatment response are correlated, the response was assessed by 2 different approaches. First, we evaluated the effect of pretreatment growth rate on the relative volume changes after GKRS. Therefore, relative volume changes, with respect to the treatment volume, were determined on the 6-month, 1-year, 2-year, and 3-year follow-up MR images. We calculated Spearman's correlation coefficients and corresponding confidence intervals using bootstrapping to investigate whether a correlation exists between the pretreatment growth rate and the volumetric treatment response.

Second, the effect of the pretreatment growth rate on the response to GKRS in terms of tumor control was evaluated. By stratifying the patient cohort using the median VDT, a comparison of Kaplan-Meier curves was made between fast-growing tumors and slow-growing tumors using the log-rank test. The characteristics that could potentially influence the outcome were assessed in both groups and tested for statistical significance. These included patient age, tumor volumes at treatment, dose-volume histograms, number of isocenters, beam-on times, and Paddick conformity indices. The Kaplan-Meier analysis can be extended to multiple stratifications, evaluating whether a linear trend is present. However, due to the limited number of failures, the possible number of stratifications is also limited. We therefore split the complete cohort into 3 groups, using the 33rd and 67th percentile values of the VDT. If a linear trend is present, it indicates a distinct relation between the VDT and the GKRS treatment outcome in terms of tumor control.

Alternatively, we evaluated the effect of the pretreatment growth rate on tumor control using a Cox proportional hazard regression analysis. Univariate analyses are employed to evaluate the influence of each of the independent variables to the treatment outcome. Apart from the pretreatment growth rate, these also included the aforementioned characteristics.

All statistical analyses were performed using IBM SPSS statistics for Windows (version 23, IBM Corp.).

\section{Results}

\section{Patient Cohort}

In our Gamma Knife center, 736 patients with sporadic unilateral VS were treated between 2002 and 2014. Of these 736 patients, 440 showed confirmed radiological progression of the tumor prior to treatment. After the records were reviewed, 129 patients $(29.3 \%)$ were excluded from this study (Fig. 1). Reasons for exclusion were less than 2 years of follow-up (22 patients, 5.0\%), pretreatment MR images not obtainable or unavailable (79 patients, $18.0 \%$ ), pretreatment MR images of poor quality (15 patients, 3.4\%), or incompatible for volumetric measurements in GammaPlan due to external MRI formats such as nonsquare images (13 patients, 3.0\%). This resulted in a patient cohort of 311 patients.

In this cohort, the median time between treatment and the available pretreatment scan was 19 months. For the

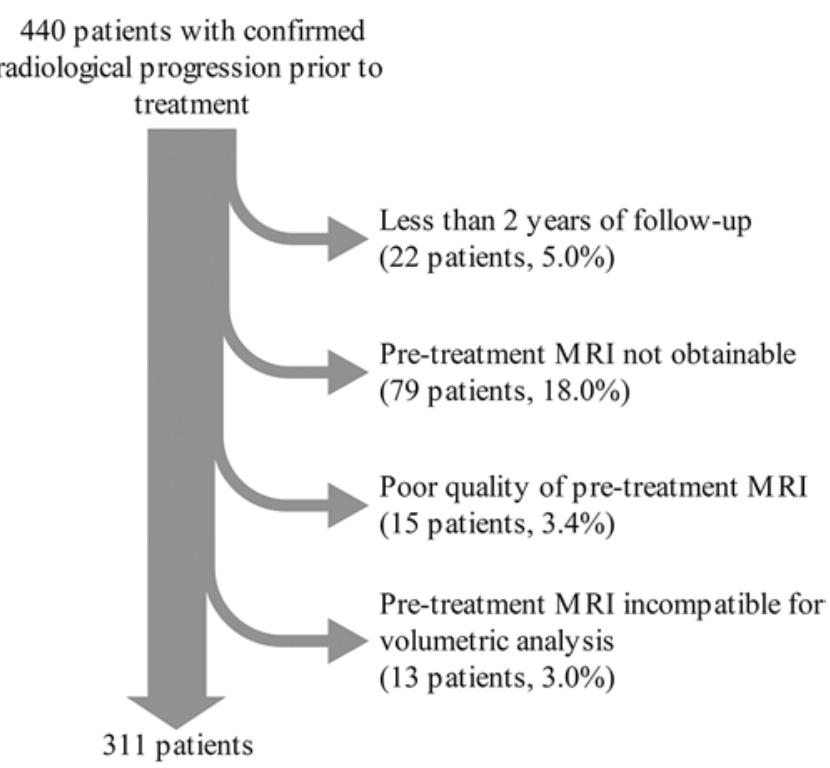

FIG. 1. Patient exclusion criteria and their resulting numbers.

VDT calculations, we used 165 T1-weighted and 146 T2weighted pretreatment MR images with a median slice thickness of $1 \mathrm{~mm}$. The resulting VDT values for the cohort had a median of 15 months with a range of 3 to 344 months. Patient and treatment-related characteristics are given in Table 1. The median posttreatment follow-up time was 60 months with a median time between 2 consecutive scans of 12 months. Lack of tumor control was observed in 35 cases (11.3\%) within this cohort, resulting in the Kaplan-Meier curve depicted in Fig. 2. One tumor exhibited obvious and excessive growth during the first 2 years after treatment, such that intervention was considered necessary. The calculated 5- and 10-year control rates of the cohort were $91.6 \%$ and $77.5 \%$, respectively.

\section{Correlation Between Pretreatment Growth Rate and GKRS Response}

To evaluate the relationship between pretreatment

TABLE 1. Patient and treatment-related characteristics

\begin{tabular}{lccc}
\hline \multicolumn{1}{c}{ Characteristic } & Median & IQR & Range \\
\hline Patient age at treatment, yrs & 59 & $51-68$ & $24-85$ \\
\hline Tumor vol at treatment, $\mathrm{cm}^{3}$ & 1.16 & $0.62-2.54$ & $0.06-12.18$ \\
\hline Pretreatment observation time, mos & 19 & $14-30$ & $6-105$ \\
\hline Posttreatment follow-up time, mos & 60 & $38-86$ & $19-159$ \\
\hline VDT, mos & 15 & $10-26$ & $3-344$ \\
\hline Prescription isodose line, \% & 55 & $47-64$ & $37-100$ \\
\hline Dose to 99\% of tumor vol, Gy & 11.9 & $11.5-13.0$ & $9.5-13.6$ \\
\hline No. of isocenters & 13 & $9-19$ & $1-43$ \\
\hline Beam-on time, mins & 41.5 & $30.9-54.7$ & $8.3-112.0$ \\
\hline Gradient index & 2.92 & $2.68-3.30$ & $2.47-6.74$ \\
\hline Selectivity & 0.87 & $0.81-0.93$ & $0.50-0.99$ \\
\hline Paddick conformity index & 0.82 & $0.77-0.85$ & $0.46-1.31$ \\
\hline
\end{tabular}




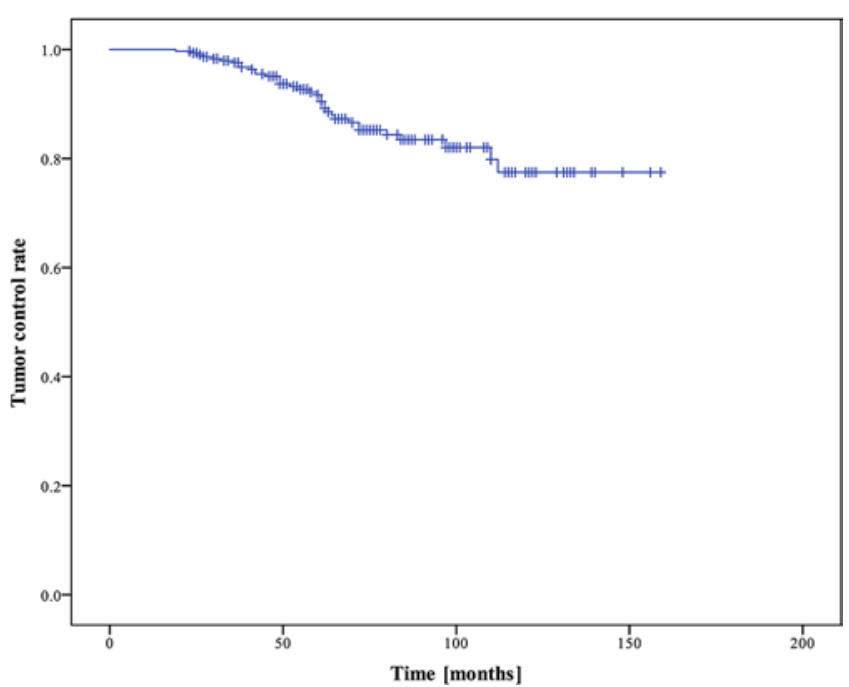

FIG. 2. Kaplan-Meier curve for the complete cohort. The calculated 5and 10 -year control rates for this cohort are $91.6 \%$ and $77.5 \%$, respectively. Tick marks indicate censored cases. Figure is available in color online only.

growth rates and volumetric tumor responses after GKRS, the relative posttreatment volume changes of each tumor were calculated based on the 6-month, 1-year, 2-year, and 3 -year follow-up MR images. The medians and interquartile ranges of these relative volume changes are given in Table 2. Correlations between the relative volume changes and the VDT were determined according to Spearman's rho method. The relative volume changes were not significantly correlated with the pretreatment growth rate.

We also investigated the effect of the VDT on the loss of tumor control after GKRS. For the Kaplan-Meier survival analysis, the patient cohort was stratified into 2 groups, a slow- and a fast-growing tumor group, with a median VDT of 15 months as the separating value; ties $(\mathrm{VDT}=15)$ were assigned to the slow-growing cohort. This stratification resulted in slow- and fast-growing tumor cohorts of 162 and 149 patients, respectively. These 2 cohorts included 10 and 25 cases of treatment failure, respectively. This difference was statistically significant (Fisher's exact test, $\mathrm{p}=0.004$ ). The median times to loss of tumor control in both groups were 63 and 49 months for the slow- and fast-growing cohorts, respectively. This difference was also statistically significant (Mann-Whitney U-test, $\mathrm{p}=0.041)$. The Kaplan-Meier curves for both cohorts are depicted in Fig. 3 left. A comparison of these curves indicates a significant difference between tumor control rates of the cohorts using a log-rank test $(\mathrm{p}=$ 0.001 ). The calculated 5- and 10-year tumor control rates were $97.3 \%$ and $86.0 \%$ in the slow-growing cohort, and $85.5 \%$ and $67.6 \%$ in the fast-growing cohort, respectively. To evaluate if characteristics other than the VDT can explain this difference, we investigated possible distinctions in the main characteristics between these 2 groups. Statistical analysis indicated that there were no significant differences between the groups (Table 3).

We performed the same Kaplan-Meier analysis using 3 groups by creating a slow-growing, an average-growing,
TABLE 2. Statistics of the relative volume changes after GKRS

\begin{tabular}{lccc}
\hline \multicolumn{1}{c}{ FU } & No. of Pts & Median (IQR) Relative Vol Change, \%* & p Value† \\
\hline 6 mos & 54 & $-4.4(-25.4$ to 13.5$)$ & 0.836 \\
\hline $1 \mathrm{yr}$ & 282 & $10.6(-11.9$ to 34.3$)$ & 0.452 \\
\hline $2 \mathrm{yrs}$ & 225 & $28.2(8.3$ to 43.5$)$ & 0.073 \\
\hline $3 \mathrm{yrs}$ & 170 & $19.6(3.1$ to 37.5$)$ & 0.173 \\
\hline
\end{tabular}

FU = follow-up; Pts = patients.

${ }^{*}$ A negative volume change denotes an increase in tumor volume. $†$ Calculated Spearman's correlation coefficients between the VDT and the relative volume changes.

and a fast-growing tumor cohort. Separation was performed by splitting the cohort according to the 33rd and 67th percentiles, where ties were assigned to the slowergrowing tumor cohort. This resulted in 3 cohorts containing 106, 108, and 97 patients with 5, 12, and 18 cases of treatment failure, respectively. The calculated 5- and 10year tumor control rates were $98.8 \%$ and $91.4 \%$ for the slow-growing cohort, $90.6 \%$ and $70.7 \%$ for the averagegrowing cohort, and $84.8 \%$ and $66.4 \%$ for the fast-growing cohort, respectively. The resulting curves are presented in Fig. 3 right, where a linear trend can be observed (logrank test, $\mathrm{p}=0.001$ ).

\section{Prediction Model of the GKRS Treatment Response}

Finally, we also investigated the effect of the main characteristics on the tumor control rates by implementing univariate Cox regression analyses. The results showed that only the VDT had a significant effect $(\mathrm{p}=0.045)$. None of the other patient and treatment-related characteristics, shown in Table 3, displayed a statistically significant influence. Consequently, no multivariate analysis was necessary. The impact of the VDT on the proportional hazards ratio is given by $\exp (\mathrm{B})$, which equals 0.970 . This means that the risk of loss of tumor control for a tumor with a given VDT will decrease with factor 0.970 for a tumor for which the VDT is 1 month greater, i.e., a slower-growing tumor. For example, the 5-year loss of tumor control in the Kaplan-Meier analysis was $8.4 \%$ in the patient cohort for a tumor with a median VDT of 15 months. If the VDT increases by 12 months, the estimated 5-year loss of tumor control rate will be $5.8 \%$.

\section{Robustness of Our Findings}

As our study mainly uses tumor volumes, the impact of the inter- and intraobserver inconsistencies of the volumetric assessments may be significant on the results. These inconsistencies are more critical in small tumors, as relative errors become larger for decreasing volumes. ${ }^{18} \mathrm{We}$ have adopted a threshold on the minimum time required between scans in the VDT calculations. However, we did not impose a threshold on the minimum tumor volume required, such that the impact of the relative volume errors is reduced. Hence, for small tumors the calculated VDT can be inaccurate. If we remove tumors smaller than $0.25 \mathrm{~cm}^{3}$ in our cohort and redo the analyses, we observe a statistically improved result from the univariate Cox regression (286 patients, $\mathrm{p}=0.012$ ). 

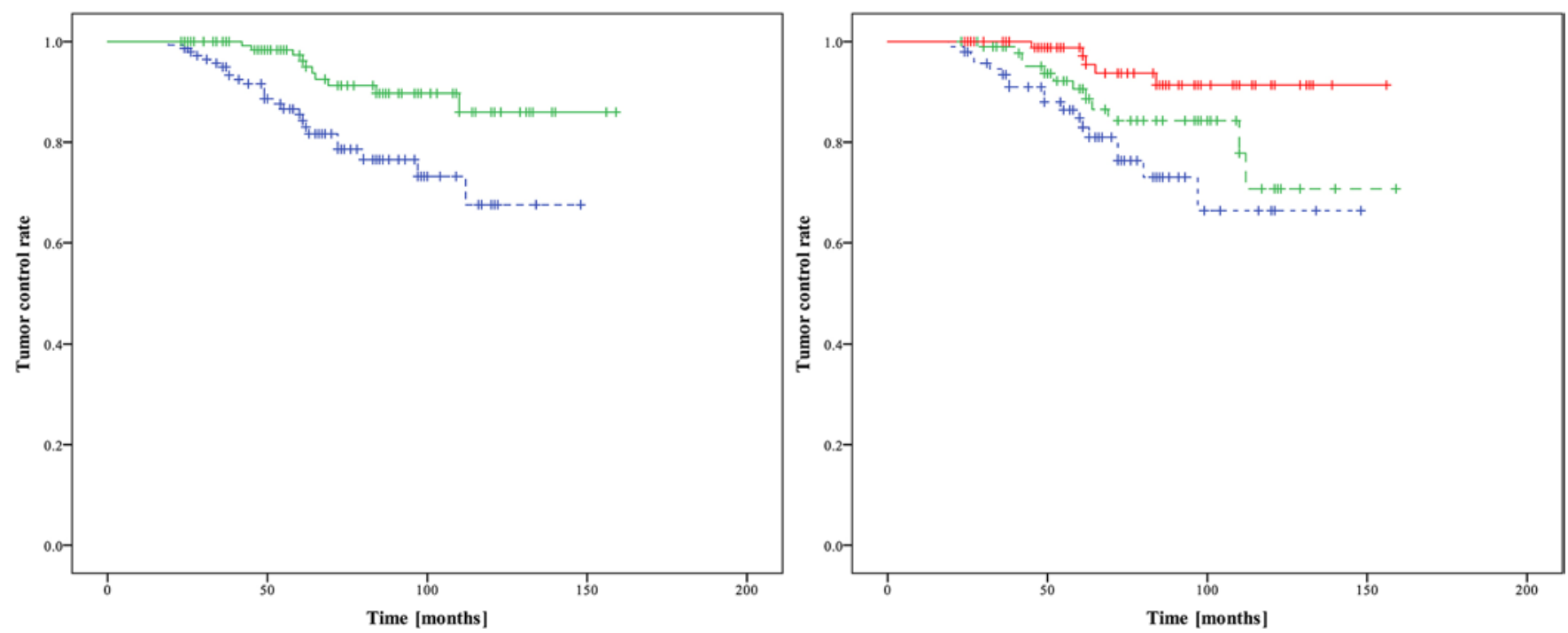

FIG. 3. Left: Kaplan-Meier curves for the fast-growing (blue dashed line) and slow-growing (green continuous line) cohorts, stratified at the median of the pretreatment growth rate. Right: Kaplan-Meier curves of the fast-growing (blue short dashes), averagegrowing (green long dashes), and slow-growing (red continuous line) cohorts, stratified by the 33rd and 67th percentiles of the pretreatment growth rate. In both graphs tick marks indicate censored cases. Figure is available in color online only.

Furthermore, the inter- and intraobserver inconsistencies also have an impact on our strict criterion concerning the treatment failure. If we redo the analyses without the so-called volumetric failures and only consider failures determined in the clinical setting, we still find a significant difference between the slow- and fast-growing tumor cohorts (Fisher's exact test, $\mathrm{p}=0.040$; Kaplan-Meier logrank test, $\mathrm{p}=0.021)$.

\section{Discussion}

This study was designed to investigate the relationship between the pretreatment growth rate and the Gamma Knife radiosurgical efficacy. This study is relevant because if the growth rate prior to treatment influences the radiosurgical outcome, the question arises whether this justifies alterations in treatment management.

The only way to accurately determine the influence of the pretreatment growth rate to the GKRS treatment outcome is to study a cohort with quantifiable tumor progression prior to treatment. We used the VDT model proposed by Varughese et al. to quantify tumor progression. ${ }^{20}$ As VSs do not necessarily grow at a regular rate, the VDT might not be a perfect fit for each individual case. However, we do not have the data to observe the growth of each individual tumor over a long period of time. Many tumors are treated when tumor growth is observed between 2 consecutive scans. With the pretreatment data available in this study, the VDT is the most accurate way to describe VS growth, as Varughese et al. concluded in their article, which specifically addresses the issue of determining growth rates of VSs.

Various previous studies have addressed the potential role of pretreatment growth rates on GKRS treatment responses, but the results are conflicting. ${ }^{8,9,14,17,21}$ In our opinion, the reported inconsistent results can be explained by methodological shortcomings in these studies. Loss of tumor control is observed rarely and often occurs several years after treatment. This fact makes it mandatory that studies on this topic include large patient numbers and long follow-up times. All 5 aforementioned studies reported on a relatively low number of patients, and only 2 studies had median follow-up times significantly larger than the generally accepted time period for pseudoprogression (Table 4). Furthermore, it is important to apply volumetric mea-

TABLE 3. Comparison of patient- and treatment-related characteristics between the fast- and slow-growing cohorts

\begin{tabular}{llll}
\hline & \multicolumn{2}{c}{ Median (IQR) } & \\
\cline { 2 - 3 } Characteristic & $\begin{array}{c}\text { Fast-Growing } \\
\text { Cohort }\end{array}$ & $\begin{array}{c}\text { Slow-Growing } \\
\text { Cohort }\end{array}$ & $\begin{array}{c}\text { p } \\
\text { Value }\end{array}$ \\
\hline $\begin{array}{c}\text { Pt age at treatment, } \\
\text { yrs }\end{array}$ & $60(49-68)$ & $59(52-67)$ & 0.866 \\
\hline $\begin{array}{c}\text { Tumor vol at treat- } \\
\text { ment, cm }\end{array}$ & $1.06(0.65-2.74)$ & $1.27(0.53-2.44)$ & 0.667 \\
\hline $\begin{array}{c}\text { Prescription isodose } \\
\text { line, \% }\end{array}$ & $55(46-63)$ & $53(48-64)$ & 0.537 \\
\hline $\begin{array}{c}\text { Dose to 100\% of } \\
\text { tumor vol, Gy }\end{array}$ & $11.4(11.1-12.2)$ & $11.4(11.0-12.0)$ & 0.089 \\
\hline $\begin{array}{c}\text { Dose to 99\% of } \\
\text { tumor vol, Gy }\end{array}$ & $12.0(11.6-13.0)$ & $11.9(11.4-12.7)$ & 0.108 \\
\hline $\begin{array}{c}\text { Dose to 95\% of } \\
\text { tumor vol, Gy }\end{array}$ & $12.6(12.4-13.8)$ & $12.6(12.3-13.8)$ & 0.083 \\
\hline No. of isocenters & $13(10-19)$ & $14(9-19)$ & 0.707 \\
\hline $\begin{array}{c}\text { Beam-on time, mins } \\
\text { Gradient index }\end{array}$ & $41.9(32.1-55.2)$ & $41.5(29.7-54.5)$ & 0.397 \\
\hline Selectivity & $2.92(2.70-3.26)$ & $2.92(2.68-3.37)$ & 0.987 \\
\hline $\begin{array}{c}\text { Paddick conformity } \\
\text { index }\end{array}$ & $0.88(0.82-0.94)$ & $0.87(0.80-0.92)$ & 0.288 \\
\hline
\end{tabular}


TABLE 4. Differences in methodological definitions and results between the different studies evaluating the relation between pretreatment growth rate and GKRS treatment outcome

\begin{tabular}{|c|c|c|c|c|c|}
\hline Authors \& Year & $\begin{array}{l}\text { No. of Pts } \\
\text { (failures) }\end{array}$ & $\begin{array}{l}\text { FU Time } \\
\text { (mos) }\end{array}$ & $\begin{array}{l}\text { Volumetric Tumor } \\
\text { Measurements }\end{array}$ & Definition of Treatment Failure & $\begin{array}{l}\text { Pretreatment Growth } \\
\text { Rate as Predictor }\end{array}$ \\
\hline Varughese et al., $2012^{21}$ & $45(13)$ & Mean 50 & Yes & Post-GKRS VDT >0 & No \\
\hline Marston et al., 2017 & $68(9)$ & Median 43.5 & No & Diameter increase $>2 \mathrm{~mm}$ & Yes \\
\hline Niu et al., 2014 & $58(3)$ & Median 27.5 & Yes & Expansion w/ homogeneous enhancement on MRI & Yes \\
\hline Timmer et al., 2011 & $67(5)$ & Mean 26 & No & Secondary treatment needed & No \\
\hline Larjani et al., 2014 & $63(7)$ & Median 32 & Yes & $\begin{array}{l}\text { No stabilization or regression of significant growth } \\
\text { at } 24 \text { mos after treatment }\end{array}$ & No \\
\hline Present study & $311(35)$ & Median 60 & Yes & Secondary treatment needed + volumetric model & Yes \\
\hline
\end{tabular}

surements. Subtle loss of tumor control can go unnoticed when obtaining linear measurements. Such small changes may be irrelevant from a clinical perspective, i.e., not demanding an intervention. However, from a scientific viewpoint, it is important to identify all cases of tumor growth after radiosurgery. It allows for an accurate assessment of the correlation between the pretreatment growth rate and the volumetric GKRS treatment response. Three of the 5 studies addressing the influence of pretreatment growth rates on the treatment outcome utilized pretreatment volumetric measurements. One study, by Timmer et al., did not exploit the actual linear pretreatment measurements, but it stratified the cohort accordingly, i.e., into growing and nongrowing tumor cohorts. ${ }^{17}$ Furthermore, there are also inconsistencies among the definitions of treatment failure (Table 4).

Therefore, in our research we have used volumetric tumor measurements in a large patient cohort with long follow-up times (Table 4). Our data clearly illustrate that the pretreatment growth rate correlated with the radiosurgical efficacy. Slow-growing tumors in our cohort are more likely to exhibit tumor control than their fast-growing counterparts. Using Kaplan-Meier analysis, the estimated 5- and 10-year tumor control rates are 97.3\% and $86.0 \%$ for the slow-growing tumors, and $85.5 \%$ and $67.6 \%$ for the fast-growing tumors, respectively. This effect was also apparent if we stratified the data of this patient cohort into 3 groups, with the most failures in the fastest-growing cohort $(\mathrm{n}=18)$, and an intermediate number of failures in the middle cohort $(n=12)$. This suggests a distinct effect of the pretreatment growth rate on tumor control after GKRS. Indeed, the Cox regression analysis was significant for the pretreatment growth rate expressed as the VDT ( $p$ $=0.045)$. In the resulting prediction model, the impact of the VDT on the risk of loss of tumor control equals a factor of 0.970 . This means that increasing the VDT by 1 month results in a decrease with a factor of 0.970 of failure risk at a certain time. It is interesting to use this factor to evaluate the risk of loss of tumor control for various cases. Some examples of this evaluation can be found in Table 5 .

It has been suggested that a higher rate of loss of tumor control after radiosurgery for fast-growing VSs can be explained by the radiobiological effect of slowing down the growth curve: fast-growing tumors will be slowed down by radiosurgery, but possibly not enough to obtain tumor control, whereas the growth curve of slow-growing tumors is bent sufficiently to obtain tumor control. ${ }^{9,16}$ However, our data indicate that relative posttreatment volume changes do not demonstrate differences between fast- and slow-growing tumors. We therefore hypothesize that the intrinsic tumor biology of fast-growing tumors makes them more likely to start growing again several years after radiosurgery, rather than radiosurgery slowing down their growth rate. However, the radiobiological effect of radiosurgery on VS remains unclear from existing literature. An ongoing discussion is whether the radiosurgical response of VS results from direct cytotoxic effects to cells, or whether it reflects indirect effects. In the review by Yeung et al., the authors discussed 3 possible mechanisms that could explain the decreased tumor control rates in certain VS tumors: the Merlin-induced imbalance in the c-Jun N-terminal kinase pathway and extracellular signalrelated kinase pathway, the inadequate angiogenesis and hypoxia, and the radioresistance during cell cycle. ${ }^{24}$ In general, cell survival data have demonstrated that cells are most sensitive to irradiation during mitosis and in the $\mathrm{G}_{2}$-phase, less sensitive in the $\mathrm{G}_{1}$-phase, and least sensitive during the S-phase. ${ }^{15}$ This would indicate that radioresistance increases for tumors that relatively lack cell division, i.e., are slow growing. However, we observed the opposite: fast-growing VSs tend to respond less to radiosurgery. This would imply that either fast-growing tumors have a superior DNA repair system ${ }^{24}$ or the response to radiosurgery reflects indirect radiation effects, such as decreasing tumor vascularity. ${ }^{25}$

The results in this study clearly indicate that the pretreatment growth rate influences the volumetric outcome of VS after GKRS. The prediction model obtained in this research provides the opportunity to determine the risk of treatment failure for each specific VS, employing the VDT. However, this prediction model needs to be validated in other cohorts.

Nevertheless, the findings of this study raise the question of whether fast-growing tumors should be treated differently, i.e., with a higher radiation dose, with microsurgery, or by reducing the radioresistance of these tumors, employing radiosensitizers. ${ }^{4,22,25,26}$ A prospective study should be designed to investigate whether an increased radiation dose or a combination of radiosensitizers with GKRS for fast-growing VS increases the overall tumor control rates, without increasing toxicity. However, $\mathrm{Fu}$ et al. recently showed that re-treatment of VS by GKRS ap- 
TABLE 5. Predicted hazard rates for loss of tumor control calculated by the prediction model created in this research

\begin{tabular}{ccc}
\hline VDT (yrs) & 5-Yr Hazard Rate & 10-Yr Hazard Rate \\
\hline 0.5 & $11.0 \%$ & $29.6 \%$ \\
\hline 1 & $9.2 \%$ & $24.7 \%$ \\
\hline 2 & $6.4 \%$ & $17.1 \%$ \\
\hline 3 & $4.4 \%$ & $11.9 \%$ \\
\hline 4 & $3.1 \%$ & $8.2 \%$ \\
\hline
\end{tabular}

These hazards are calculated including the volumetric failure definition.

pears to be an effective strategy, suggesting that fast-growing tumors may benefit from a second GKRS treatment. ${ }^{3}$

Another possible benefit of being able to predict the chance of tumor control may be the personalization of the standard follow-up protocol: for patients with a slow-growing VS, the follow-up interval may be extended, while for fast-growing VS, patients may be monitored more closely.

\section{Limitations}

One of the most important limitations of this study, as well as other studies on this topic, is that there is no clear consensus on the explicit criteria for treatment failure after GKRS. Hence, direct comparison of tumor control rates reported in various studies is problematic, as the definition of treatment failure appears to be inconsistent. ${ }^{6,9,16}$ Some studies define treatment failure as the requirement for microsurgical resection and do not mention whether a second GKRS treatment is considered as failure. ${ }^{1,2}$ Others, like the radiosurgical team at our center, define tumor control as the absence of radiologically identified progression, which is usually done by linear assessment. ${ }^{12,13,23}$ However, even with proven tumor progression, intervention may still be undesirable. Therefore, in addition to proven tumor progression followed by intervention, we have employed a mathematical model for determining treatment failure to simultaneously account for missed small progressions in the clinical setting and undesirable interventions. This model may not be clinically relevant, but it provides an objective measure for determining treatment failure. Because of this strict criterion, the results of our Kaplan-Meier analysis display lower tumor control rates than those reported in other studies.

Furthermore, the determination of tumor control is troublesome, because of the pseudoprogression phenomenon. Most tumors presenting with pseudoprogression reach a maximum volume after a median of 5 months and first signs of regression at a median of 15 months. . $^{511,17,19}$ In this study, we tried to circumvent this issue in defining loss of tumor control by only considering volumetric measurements beyond 2 years after treatment. However, it is claimed that pseudoprogression can occur as late as 3-4 years after radiosurgery. ${ }^{16}$

Another limitation of this research is that the data are retrospectively analyzed. This leads, for instance, to uncertainties in the volumetric assessment of the tumors, due to differences in MRI scans. For 146 patients, the T1weighted contrast-enhanced MRI study obtained prior to treatment was unavailable or of poor quality. Employing the T2-weighted MR images for these patients may have introduced uncertainties in the determined tumor volumes. This effect is more critical in small tumors, as relative errors in the volumetric assessments become larger for decreasing volumes. ${ }^{18}$ However, we have shown that excluding these small tumors results in statically improved results.

\section{Conclusions}

In current literature, the influence of the pretreatment growth rate of VS on the GKRS treatment response can be classified as undetermined, due to the conflicting results reported by various studies. Methodological imperfections can possibly explain these contradicting results. Because of the large number of patients and the long follow-up times of our cohort and the volumetric tumor assessments both prior to and after GKRS treatment, we had the unique opportunity to accurately investigate the influence of the pretreatment tumor growth rate on the volumetric GKRS treatment response. We have found a predictive model illustrating the negative influence of the pretreatment VS growth rate on the radiosurgical treatment efficacy. The resulting tumor control rates confirm the high efficacy of GKRS for slow-growing VS. However, the fast-growing tumors exhibited significantly lower control rates. Our analyses demonstrate that for these tumors, the calculated 5- and 10 -year tumor control rates are $85.5 \%$ and $67.6 \%$, respectively. For these cases, different treatment strategies may be considered. Additionally, the results of this research may help in patient counseling and in determining a patient-specific follow-up protocol, where the follow-up frequency of slow-growing tumors may be reduced with respect to the frequency needed for fast-growing tumors.

\section{Acknowledgments}

This research is funded by ZonMw, dossier no. 80-84200-9815222, funds received by Patrick P. J. H. Langenhuizen.

\section{References}

1. Chopra R, Kondziolka D, Niranjan A, Lunsford LD, Flickinger JC: Long-term follow-up of acoustic schwannoma radiosurgery with marginal tumor doses of 12 to 13 Gy. Int J Radiat Oncol Biol Phys 68:845-851, 2007

2. Flickinger JC, Kondziolka D, Niranjan A, Maitz A, Voynov G, Lunsford LD: Acoustic neuroma radiosurgery with marginal tumor doses of 12 to 13 Gy. Int J Radiat Oncol Biol Phys 60:225-230, 2004

3. Fu VX, Verheul JB, Beute GN, Leenstra S, Kunst HPM, Mulder JJS, et al: Retreatment of vestibular schwannoma with Gamma Knife radiosurgery: clinical outcome, tumor control, and review of literature. J Neurosurg 129:137-145, 2018

4. Hastak K, Bhutra S, Parry R, Ford JM: Poly (ADP-ribose) polymerase inhibitor, an effective radiosensitizer in lung and pancreatic cancers. Oncotarget 8:26344-26355, 2017

5. Hayhurst C, Zadeh G: Tumor pseudoprogression following radiosurgery for vestibular schwannoma. Neuro Oncol 14:87-92, 2012

6. Klijn S, Verheul JB, Beute GN, Leenstra S, Mulder JJS, Kunst HPM, et al: Gamma Knife radiosurgery for vestibular schwannomas: evaluation of tumor control and its predictors in a large patient cohort in The Netherlands. J Neurosurg 124:1619-1626, 2016 
7. Kondziolka D, Wolf A: Commentary: ten-year follow-up on tumor growth and hearing in patients observed with an intracanalicular vestibular schwannoma. Neurosurgery 80:57-59, 2017

8. Larjani S, Monsalves E, Pebdani H, Krischek B, Gentili F, Cusimano $\mathrm{M}$, et al: Identifying predictors of early growth response and adverse radiation effects of vestibular schwannomas to radiosurgery. PLoS One 9:e110823, 2014

9. Marston AP, Jacob JT, Carlson ML, Pollock BE, Driscoll CLW, Link MJ: Pretreatment growth rate as a predictor of tumor control following Gamma Knife radiosurgery for sporadic vestibular schwannoma. J Neurosurg 127:380-387, 2017

10. Miller T, Lau T, Vasan R, Danner C, Youssef AS, van Loveren $\mathrm{H}$, et al: Reporting success rates in the treatment of vestibular schwannomas: are we accounting for the natural history? J Clin Neurosci 21:914-918, 2014

11. Mindermann T, Schlegel I: How to distinguish tumor growth from transient expansion of vestibular schwannomas following Gamma Knife radiosurgery. Acta Neurochir (Wien) 156:1121-1123, 2014

12. Myrseth E, Møller P, Pedersen PH, Lund-Johansen M: Vestibular schwannoma: surgery or gamma knife radiosurgery? A prospective, nonrandomized study. Neurosurgery 64:654-663, 2009

13. Nakaya K, Niranjan A, Kondziolka D, Kano H, Khan AA, Nettel B, et al: Gamma Knife radiosurgery for benign tumors with symptoms from brainstem compression. Int J Radiat Oncol Biol Phys 77:988-995, 2010

14. Niu NN, Niemierko A, Larvie M, Curtin H, Loeffler JS, McKenna MJ, et al: Pretreatment growth rate predicts radiation response in vestibular schwannomas. Int J Radiat Oncol Biol Phys 89:113-119, 2014

15. Pawlik TM, Keyomarsi K: Role of cell cycle in mediating sensitivity to radiotherapy. Int J Radiat Oncol Biol Phys 59:928-942, 2004

16. Régis J, Delsanti C, Roche PH: Editorial. Vestibular schwannoma radiosurgery: progression or pseudoprogression? J Neurosurg 127:374-379, 2017

17. Timmer FCA, Mulder JJS, Hanssens PEJ, van Overbeeke JJ, Donders RT, Cremers CWRJ, et al: Gamma knife radiosurgery for vestibular schwannomas: identification of predictors for continued tumor growth and the influence of documented tumor growth preceding radiation treatment. Laryngoscope 121:1834-1838, 2011

18. van de Langenberg R, de Bondt BJ, Nelemans PJ, Baumert BG, Stokroos RJ: Follow-up assessment of vestibular schwannomas: volume quantification versus two-dimensional measurements. Neuroradiology 51:517-524, 2009

19. van de Langenberg R, Dohmen AJC, de Bondt BJ, Nelemans PJ, Baumert BG, Stokroos RJ: Volume changes after stereotactic LINAC radiotherapy in vestibular schwannoma: control rate and growth patterns. Int J Radiat Oncol Biol Phys 84:343-349, 2012

20. Varughese JK, Breivik CN, Wentzel-Larsen T, Lund-Johan- sen M: Growth of untreated vestibular schwannoma: a prospective study. J Neurosurg 116:706-712, 2012

21. Varughese JK, Wentzel-Larsen T, Pedersen PH, Mahesparan R, Lund-Johansen M: Gamma knife treatment of growing vestibular schwannoma in Norway: a prospective study. Int J Radiat Oncol Biol Phys 84:e161-e166, 2012

22. Wang $\mathrm{H}, \mathrm{Mu} \mathrm{X}, \mathrm{He} \mathrm{H}$, Zhang XD: Cancer radiosensitizers. Trends Pharmacol Sci 39:24-48, 2018

23. Wangerid T, Bartek J Jr, Svensson M, Förander P: Long-term quality of life and tumour control following gamma knife radiosurgery for vestibular schwannoma. Acta Neurochir (Wien) 156:389-396, 2014

24. Yeung AH, Sughrue ME, Kane AJ, Tihan T, Cheung SW, Parsa AT: Radiobiology of vestibular schwannomas: mechanisms of radioresistance and potential targets for therapeutic sensitization. Neurosurg Focus 27(6):E2, 2009

25. Yue WY, Clark JJ, Telisak M, Hansen MR: Inhibition of c-Jun N-terminal kinase activity enhances vestibular schwannoma cell sensitivity to $\gamma$ irradiation. Neurosurgery 73:506516,2013

26. Zhu L, Wu K, Ma S, Zhang S: HDAC inhibitors: a new radiosensitizer for non-small-cell lung cancer. Tumori 101:257262,2015

\section{Disclosures}

The authors report no conflict of interest concerning the materials or methods used in this study or the findings specified in this paper.

\section{Author Contributions}

Conception and design: Langenhuizen, Zinger, Leenstra, Verheul. Acquisition of data: Langenhuizen, Verheul. Analysis and interpretation of data: Langenhuizen, Verheul. Drafting the article: Langenhuizen, Verheul. Critically revising the article: Zinger, Hanssens, Kunst, Mulder, Leenstra, de With, Verheul. Reviewed submitted version of manuscript: Zinger, Hanssens, Kunst, Mulder, Leenstra, de With, Verheul. Approved the final version of the manuscript on behalf of all authors: Langenhuizen. Statistical analysis: Langenhuizen, Verheul. Administrative/technical/material support: Zinger, Hanssens, Kunst, Mulder, Leenstra, de With, Verheul. Study supervision: Zinger, Leenstra, de With, Verheul.

\section{Supplemental Information}

Previous Presentations

Preliminary results of this study were presented as an oral presentation at the 13th International Stereotactic Radiosurgery Society Congress, Montreux, Switzerland, May 28-June 1, 2017.

\section{Correspondence}

Patrick P. J. H. Langenhuizen: ETZ Hospital, Tilburg, The Netherlands.p.langenhuizen@etz.nl. 